Case Report

\title{
Therapy and Health System-Related Factors Influencing Adherence to ART Treatment among HIVIAIDS Patients in Embu Teaching and Referral Hospital Comprehensive Care Clinic
}

\author{
Evangeline Kananu Njue Mugoh ${ }^{1, *}$, Ephantus Kabiru', Joyce Mwaniki \\ ${ }^{1}$ Department of Community Health, Kenyatta University, Nairobi, Kenya \\ ${ }^{2}$ Department of Plant Sciences, Kenyatta University, Nairobi, Kenya \\ Email address: \\ evangelinemugoh@gmail.com (E. K. N. Mugoh), ewkabiru@yahoo.com (E. Kabiru), joycemwaniki@gmail.com (J. Mwaniki) \\ ${ }^{*}$ Corresponding author
}

To cite this article:

Evangeline Kananu Njue Mugoh, Ephantus Kabiru, Joyce Mwaniki. Therapy and Health System-Related Factors Influencing Adherence to ART Treatment Among HIV/AIDS Patients in Embu Teaching and Referral Hospital Comprehensive Care Clinic. American Journal of Nursing Science. Vol. 5, No. 5, 2016, pp. 169-174. doi: 10.11648/j.ajns.20160505.11

Received: July 26, 2016; Accepted: August 4, 2016; Published: August 21, 2016

\begin{abstract}
Adherence to antiretroviral therapy is a major predictor of the survival of individuals living with HIV/AIDS. Appropriate use of antiretroviral (ARV's) has improved the health of many human immunodeficiency virus (HIV) positive individuals. The effectiveness of HIV treatment depends on sustenance of high levels of adherence to ARV; however, ARV regimens are often complicated and can be affected by varying dosing schedules, failing to have proper dietary requirements and patients developing adverse effects. The objective of this study was to determine therapy and health system related factors influencing adherence to Anti-retroviral drugs among adult HIV/ AIDS patients in Embu County Teaching and Referral Hospital Comprehensive Care Clinic. The study applied cross sectional descriptive design with stratified random sampling used to obtain relative proportion of male and female adult respondents in the sample population. $332 \mathrm{HIV}$ positive patients were chosen from a total of 1694 patients who were active in ART for more than one year. A semi-structured interview schedules was used to obtain patients view on various dimension or ART services at the facility. ART adherence was at $48.2 \%$. There was significant association between adherence to ART treatment and the type of drug a respondent was in with AZT, ddl, NFV having the highest proportion of sub-optimal adherence to treatment while respondents on D4T, 3TC, NVP had the lowest proportion of sub-optimal adherence to treatment. Convenience of the facilities for people with chronic ailments and waiting time at the facilities were reported to be good (73.0\%) and excellent $(75.3 \%)$ respectively.
\end{abstract}

Keywords: ART Adherence, Treatment-Related Factors, Health System Factors

\section{Introduction}

\subsection{Background Information}

More than 1.5 million people were infected with HIV in Kenya by the year 2005 but in the year 2012, 1.6 million people were living with HIV with a prevalence rate of $6.1 \%[1$, 2]. Kenyan new HIV infections are estimated to have stabilized at an average of 89,000 among adults and about 11,000 among children annually. However progress has been made with HIV prevalence dropping from $10.5 \%$ in 1996 to $6.1 \%$ in 2012 although HIV continues to contribute the highest mortality rates, increase households burden and straining national health systems [2]. In the Kenya Aids Strategic Framework 2014/2015 - 2018/2019, Kenya aims at reducing new HIV infections by $75 \%$ and reduce AIDS related mortality by $25 \%$ in the next five years [3]. In addition, Kenya's Vision 2030 aims at achieving comprehensive HIV prevention, treatment and care.

Of the 1.6 million PLHIV, 656,359 were on ART against 
estimated 902,302 in 2014 (141,608 children and 760,694 adults) PLHIV needing ART according to WHO 2009 guidelines [4] while according to the Kenya AIDs Indicator Survey $2014,78.4 \%$ of adults and $42.5 \%$ of children eligible for ART coverage were receiving treatment as of year 2013 [5]. The low uptake in children was because of poor awareness on the part of parents and caregivers than non-availability of drugs. Despite this wide coverage there have been concerns with adherence to treatment $[6,7,8]$.

\subsection{Problem Statement}

Non-adherence to ART may result in regimen failure, immune suppression, emergence of resistant viral strains, limited future treatment options and higher treatment costs [9]. In low-resource settings where ART access continues to increase, the implications of non-adherence are great, as few second line regimens are available to those who develop resistance and experience treatment failure [10]. Several studies in Kenya have documented varied levels of ART adherence ranging from $17 \%$ to $91 \%[6,11,7]$. Embu County, as of 2014 , had an HIV prevalence of $3.7 \%$ with $80.8 \%$ (3718 out of 4600) of adult PLHIV eligible for ART having access to treatment [12]. However there was no documented study on the influence of treatment and health system factors on adherence levels among HIV adult patients in Embu County Teaching and Referral Hospital. Therefore this study sought to explore therapy and health system related factors that influence adherence to ART among AIDS patients in Embu

\subsection{Objectives}

i. To establish the effect of ART drugs and treatment of co-infections on adherence to ART treatment among AIDS patients.

ii. To determine how health care facilities and health care providers influence adherence to ART among AIDS patients.

\section{Literature Review}

\subsection{ART Therapy}

Any levels of adherence below 95\% have been associated with poor suppression of HIV viral load and a lowering of CD4 count leading to disease progression and development of drug resistance [13]. Evidence suggests that adherence greater than $95 \%$ is necessary to adequately suppress viral replication and produce a durable response and stop disease progression [14]. ART is a complex treatment that is characterized by pill burden, dietary, fluid restrictions and timing of medication intake. The complexity of drug regimens is one of the causes of non-adherence among patients; in addition, daily dosing regimens of taking drugs three times or more are associated with non-adherence [15]. According to a study by Curioso, et al [16], it was found that having a fixed routine and the use of reminder tools enhanced adherence to ART among patients. The pill burden is a major challenge since combination of ART is used and it often contains 2-20 pills that have to be taken in a day together with the other factors to be considered like timing of dosages and food requirements [17]. A study by Wakibi et al [7] found that $17 \%$ patients observed that ART therapy was disruptive in their life.

Although ART drugs have been provided free of charge since 2009 in Kenya, however, ART associated costs including prescription and diagnostic testing remain as cost barriers [7]. It is estimated that retention on ART treatment costing in Kenya consists 50\% ART medications while $15 \%$ consists of non-ART medications and 15\% consists of laboratory tests [18]. This shows that despite provision of free ART, patients have to foot more than $30 \%$ of the costs associated with adhering to treatment in addition to other costs such as transportation.

\subsection{Interpersonal Relationships with Health Providers}

Effective treatment relationships are characterized by an atmosphere in which alternative therapeutic means are explored, the regimen is negotiated, adherence is discussed, and follow-up is planned [19]. Cohesive partnerships and effective interpersonal communication make it possible for patients and physicians to work together to help patients follow mutually agreed-upon recommendations in addition to promoting greater patient satisfaction with medical care, which in turn fosters higher levels of adherence [20]. Positive and open relationships with their medical providers as an important adherence facilitator while also feeling comfortable to ask questions, talk about challenges, and feeling a part of decision making with one's medical provider play a key role in facilitating adherence. Achieving optimal adherence requires the commitment of both patient and provider, however, low quality of patient-provider relationship affects adherence to ART [21]. Lack of trust or dislike of a patient health care-provider, social isolation, negative publicity regarding HAART or the medical establishment, discouraging social network are impediment to ARV adherence [8].

\section{Methodology}

This was a descriptive cross sectional research that utilized quantitative research method of data collection and analysis conducted in Embu Teaching and Referral Hospital comprehensive care clinic among HIV adult that were active on ART for more than one year. A sample of 332 patients was used. Stratified sampling was used to sample patients. Data was collected using face-to-face interviews using structured questionnaires. Quality control measures were employed including pre-test, validity and reliability checks.

Authority to carry out the study was sought from NACOSTI. Approval to conduct research was sought from Kenyatta University Ethical Review Committee; and clearance from Embu Teaching and Referral Hospital and Embu County authorities. Respondents consented to participate with confirmation of confidentiality. Collected data was cross-checked for completeness and any missing entries corrected upon completion of each interview.

Data coding, entry and analysis was done using the SPSS 
version 20 software. The questionnaires were inspected to detect errors and omissions and corrected immediately. Data was analyzed using descriptives including frequencies and percentages and Chi-square tests for inferential. Findings were presented as text, graphs and tables.

\section{Findings and Discussion}

\subsection{Adherence to ART Treatment}

Both methods of evaluating adherence indicated that 160 patients had optimal adherence to treatment as shown in Table 1. However there were 11 patients which according to pill count had sub-optimal adherence to treatment while they evaluated themselves as having $100 \%$ adherence. And therefore because the pill-counts is considered the most 'objective' of the approach to assessing ART adherence, the $160(48.2 \%)$ were considered to have had optimal ART adherence and the rest having sub-optimal adherence to ART. This was different from a study by Kidder et al [22] where in a much higher figure $84.6 \%$ of 30 -days optimal adherence was reported in a longitudinal group-randomized trial in Kenya. A study done in Pumwani Hospital by Karanja [6] reported higher levels of adherence, where $91 \%$ of the respondents showed perfect adherence levels between 95 and 100\% according to hospital records as opposed to this study in Embu comprehensive care clinic. Similar findings were made among HIV patients attending Moi Teaching and Referral Hospital where it was reported that the overall adherence levels based on timing of taking ARVs was low for all respondents $43.2 \%$, clearly indicated that there were serious barriers to adherence [11]. A much lower adherence rate (17\%) was observed in two public health facilities in Nairobi [7].
Table 1. Comparison between Self-report using visual analogue and Pill Count.

\begin{tabular}{lll}
\hline $\begin{array}{l}\text { Self-report adherence in the } \\
\text { last one month using visual } \\
\text { analogue }\end{array}$ & $\begin{array}{l}\text { Adherence to ART treatment (pill } \\
\text { count) }\end{array}$ \\
\cline { 2 - 3 } & Sub-optimal & Optimal \\
\hline $10 \mathrm{~cm}$ & 11 & 160 \\
$9 \mathrm{~cm}$ & 118 & 0 \\
$8 \mathrm{~cm}$ & 31 & 0 \\
$<=7 \mathrm{~cm}$ & 12 & 0 \\
\hline
\end{tabular}

\subsection{Influence of Quality of Treatment Services and ART} Drugs on Adherence to ART

\subsubsection{Quality of Treatment Services}

The respondents reported excellent availability of ARV The respondents reported excellent availability of ARV services at the hospital/CCC (59.6\%); good TB counseling (56.3\%); excellent ART drugs counseling (56.0\%); good nutritional counseling $(52.7 \%)$ and excellent adherence counseling $(59.3 \%)$ as shown in Table 2. This showed that there was sufficient availability of quality counseling and therefore not a hindrance to ART adherence. Various studies have documented that patient education and counseling facilitated medication adherence $[10,9$, 23]. However it has been argued that availability of these counseling services in limited resource settings like Kenya is constrained by shortage of health workers which imply that the health workers are overworked hence cannot provide quality counseling [24]. A study in Ethiopia noted that some ART nurses did not have empathy and rushed when writing prescriptions, without addressing patients' concerns about their treatment [9]. Similar findings were also made in Nairobi where it was reported that when counseling services were available at early intensive adherence counseling when a patient is being started on HAART, it resulted in a significant reduction in poor adherence and virologic failure [25].

Table 2. ART Services offered in the facility.

\begin{tabular}{lllll}
\hline ART Services & Excellent & Good & Average & Poor \\
\hline Overall availability of ARV services at the CCC & $198(59.6)$ & $124(37.3)$ & $9(2.7)$ & $1(0.3)$ \\
TB counselling & $68(20.5)$ & $187(56.3)$ & $46(13.9)$ & $31(9.3)$ \\
ART drugs counselling & $186(56.0)$ & $113(34)$ & $30(9)$ & $3(0.9)$ \\
Nutritional Counselling & $115(34.6)$ & $175(52.7)$ & $40(12)$ & $2(0.6)$ \\
Adherence counselling & $197(59.3)$ & $109(32.8)$ & $24(7.2)$ & $2(0.6)$ \\
\hline
\end{tabular}

\subsubsection{Treatment of Co-infections}

As indicated in Table 3, majority of the respondents did not know about the charges for dermatological, ENT, psychiatric treatment services. However, majority of those who knew about the existence of treatment of co-infection services considered that these services were offered at higher charges except for dermatological treatment services which were mostly considered free of charge. A study in Botswana showed that adherence was higher when cost was not an obstacle and poor patients were able to achieve excellent rates of adherence when they had access to free laboratory monitoring and subsidized ART [26]. Similar findings was also made in a study in Cameroon where it was observed that although ART drugs were provided free of charge, other related services were still funded by out-of-pocket payments hence a cause of non-adherence [27].

Table 3. Treatment of co-infections charges.

\begin{tabular}{|c|c|c|c|c|c|}
\hline & High & Reasonable & Low & Don't know & Free of charge \\
\hline Dermatological treatment & $75(22.6)$ & $13(3.9)$ & $9(2.7)$ & $148(44.6)$ & $87(26.2)$ \\
\hline ENT treatment & $86(25.9)$ & $48(14.5)$ & $9(2.7)$ & $175(52.7)$ & $14(4.2)$ \\
\hline Ophthalmological treatment & $119(35.8)$ & $104(31.3)$ & $17(5.1)$ & $79(23.8)$ & $13(3.9)$ \\
\hline Psychiatric treatment & $96(28.9)$ & $72(21.7)$ & $17(5.1)$ & $141(42.5)$ & $6(1.8)$ \\
\hline
\end{tabular}




\subsubsection{Type of ART Drugs and Adherence}

Respondents on Zidovudine (AZT), Didanosure (ddl), Nelfinavir (NFV) had the highest proportion of sub-optimal adherence to treatment $4(80 \%)$ and the lowest proportion of optimal adherence $1(20 \%)$ while respondents on Stavudine (D4T), Lamivudine (3TC), Efavirenz (EFV) had the highest proportion of optimal adherence $33(66 \%)$ as shown in Figure 1. There was significant association (Chi-square p-value $=.034$ ) between adherence to ART treatment and the type of drug a respondent was in. This difference could be explained by the scheduling of different combination or the side effects associated with each combination [28]. different findings were reported in Ethiopia where relatively higher proportion of patients who missed ART was among the groups on $\mathrm{D} 4 \mathrm{~T}+3 \mathrm{TC}+\mathrm{EFV} \quad(18.2 \%) \quad$ while $14.5 \%$ missed TDF-3TC-EFVand $12.5 \%$ missed AZT-3TC- NVP [29]. Similar findings were made in Southern Malawi where it was found out that although patients are currently being transferred from the old regimen (Tenofovir) to the new regimen (Efavirenz), side effects were still a common reason their patients reported missing medication doses [30].

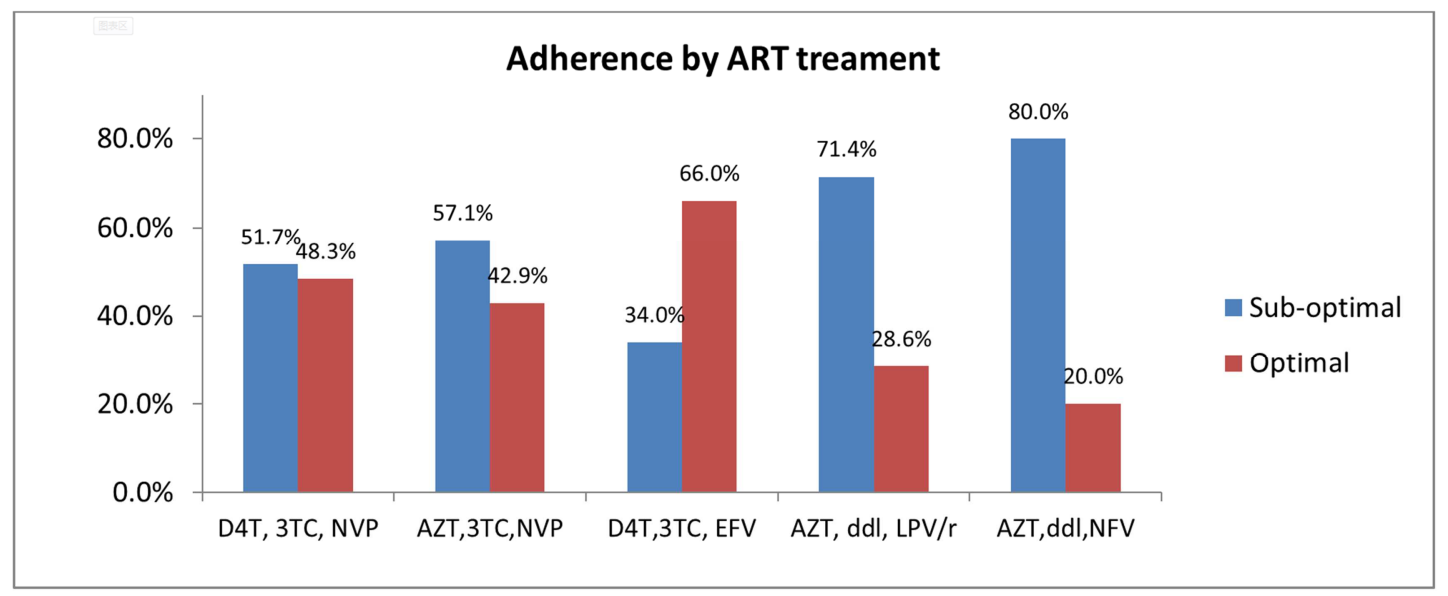

Figure 1. Adherence by ART treatment.

As shown in Table 4, accessibility to the health facility was reported to be good by 167 (50.3\%) of the respondents. Convenience of the facilities for people with chronic ailments was rated to be good by 154 (46.4\%) respondents while the waiting time at the facilities was reported to be average by 128 $(38.6 \%)$ respondents. The general state of the infrastructure at the health facility was rated to be good by 148 (44.6\%) respondents, and general cleanliness was also rated to be good $161(39.5 \%)$. However the privacy/confidentiality during examination at the facility/CCC was reported to be poor 105 (31.6\%). These findings contradicted several other studies observed that distance to treatment centre is of great concern to PLHIV and one of the key factors preventing adherence [31, 32]. This was in contradiction with other studies done in Zimbabwe by Skovdal et al [33] and Biadgilign et al [34] in Ethiopia where waiting time and opening hours were found to be a hindrance to adherence to ARV treatment.

Table 4. Health care facilities rating.

\begin{tabular}{lllll}
\hline Health care facilities rating & Excellent & Good & Average & Poor \\
\hline Physical accessibility of facility & $111(33.4)$ & $167(50.3)$ & $47(14.2)$ & $7(2.1)$ \\
Waiting time at the facility & $75(22.6)$ & $118(35.5)$ & $128(38.6)$ & $11(3.3)$ \\
Convenience of facilities for people with chronic ailments & $68(20.5)$ & $154(46.4)$ & $55(16.6)$ & $55(16.5)$ \\
Privacy/confidentiality during examination at the facility/CCC & $75(22.6)$ & $76(22.9)$ & $76(22.9)$ & $105(31.6)$ \\
General state of the infrastructures & $70(21.1)$ & $148(44.6)$ & $79(23.8)$ & $35(10.5)$ \\
General cleanliness & $161(48.5)$ & $131(39.5)$ & $39(11.7)$ & $1(0.3)$ \\
\hline
\end{tabular}

\subsubsection{Respondents' Perception of Healthcare Workers}

According to Table 5, it was revealed that accessibility to health personnel in the facility were reported to be excellent $(52.6 \%)$ while quality of time spent by doctors addressing the respondent's needs $(76.8 \%)$ were reported as excellent and provision of health education was reported as good (55.1\%). Similar findings were made by Karanja [6] where she observed that most of the Pumwani Hospital CCCs participants, $63 \%$ take less than an hour queuing before being attended by a health care provider during clinic appointments,
$73 \%$ rated the services delivered at the clinic as good, and 99\% indicated that they trust the health care providers. This finding also contradicts a study in India which indicated that up to a quarter of patients visited health centre and did not meet the counselors but just collected the drugs from pharmacy and left [35]. Similar findings were made in Zambia where HIV patients reported to have spent quality and right amount of time with healthcare workers [36]. A study in Ethiopia found out that dissatisfaction with healthcare services were major reasons for patients being non-adherent [9]. 
Table 5. Respondents' Perception of Healthcare workers.

\begin{tabular}{lllll}
\hline Healthcare workers rating & Excellent & Good & Average & Poor \\
\hline Quality of time spent by doctors & $255(76.8)$ & $59(17.8)$ & $13(3.9)$ & $5(1.5)$ \\
Accessibility of health personnel & $99(29.9)$ & $174(52.6)$ & $41(12.4)$ & $17(5.1)$ \\
Provision of health education & $183(55.1)$ & $122(36.7)$ & $23(6.9)$ & $4(1.2)$ \\
\hline
\end{tabular}

\section{Conclusions}

1. There was significant association between adherence to ART treatment and the type of drug a patient was taking with AZT, ddl, NFV having the highest proportion of sub-optimal adherence to treatment while patients on D4T, 3TC, NVP had the lowest proportion of sub-optimal adherence to treatment.

2. The attitude of the patient towards the health provider was rated excellent and the general condition of the infrastructures was found to be good however the privacy/confidentiality during examination at the facility/CCC was poor.

\section{Recommendations}

1. The study recommends that the hospital needs to improve the infrastructure so as to boost privacy in the clinic so that patients can be able to discuss their issues with clinicians without fear of being heard by other patients during service provision.

2. Finally the study recommends although that ART and TB drugs were consistently available, the hospital should ensure availability of other necessary drugs for opportunistic infections and provision of free laboratory tests for the HIV patients, taking into consideration that most of the patients socio-economic status are very low therefore making them have difficulties in affording even the cheapest treatment.

\section{Acknowledgments}

The authors of this publication "Therapy-Related and Health System-Related Factors Influencing Adherence to ART Treatment among HIV/AIDS Patients in Embu Teaching and Referral Hospital Comprehensive Care Clinic" would like to thank Kenyatta University for supervision; Embu County Teaching and Referral Hospital for permission and Exactitude Research Consultants (Ltd) for data management services.

\section{Competing Interests}

All authors declare that: there are no significant competing financial, professional or personal interests that might have influenced the performance or presentation of the work described in this manuscript.

\section{Conflict of Interest}

All authors report no conflicts of interest in this publication.

\section{Authors' Contributions}

Authors made substantial contributions to conception and design, and/or acquisition of data, and/or analysis and interpretation of data.

\section{References}

[1] UNICEF. (2013) UNICEF Kenya. [Online]. http://www.unicef.org/infobycountry/kenya_statistics.html

[2] Avert. (2015) Avert. [Online]. http://www.avert.org/professionals/hiv-around-world/sub-saha ran-africa/kenya

[3] NACC, "Kenya AIDS Strategic Framework 2014/2015 2018/2019," National AIDS Control Council, Ministry of Health, 2014.

[4] NACC, "Kenya AIDS Response Progress Report 2014: Progress towards Zero," Nairobi, 2014.

[5] NASCOP, "Kenya HIV Estimates: June 2014," Nairobi, 2014.

[6] Sarah Wangui Karanja, "Factors Influencing Adherence to Antiretroviral Medications Among Patients Living With HIV in Kenya," Department of Sociology and Social Work, University of Nairobi, Thesis 2013.

[7] Samwel N Wakibi, Zipporah W Ng'ang'a, and Gabriel G Mbugua, "Factors associated with non-adherence to highly active antiretroviral therapy in Nairobi, Kenya," AIDS Research and Therapy, vol. 8, no. 43, 2011, doi: 10.1186/1742-6405-8-43.

[8] Onyango Daniel Oloo, "Barriers To Antiretroviral Treatment Adherence Among Hiv Infected Tuberculosis Patients In Jaramogi Oginga Odinga Teaching And Referral Hospital Kisumu, Kenya," Department of Extra Mural Studies, University of Nairobi, Master's Thesis 2013.

[9] Woldesellassie M. Bezabhe et al., "Barriers and Facilitators of Adherence to Antiretroviral Drug Therapy and Retention in Care among Adult HIV-Positive Patients: A Qualitative Study from Ethiopia," PLoS One, vol. 9, no. 5, p. e97353, 2014, doi: 10.1371/journal.pone.0097353.

[10] Sarah Finocchario-Kessler et al., "Patient Communication Tools to Enhance ART Adherence Counseling in Low and High Resource Settings," Patient Education and Counseling, vol. 89, no. 1, pp. 163-170, 2012, doi: 10.1016/j.pec.2012.03.020.

[11] N. C Talam, P. Gatongi, J. Rotich, and S. Kimaiyo, "Factors Affecting Antiretroviral Drug Adherence Among HIV/AIDS Adult Patients Attending HIV/AIDS Clinic at Moi Teaching and Referral Hospital, Eldoret, Kenya.," East African Journal of Public Health, vol. 5, no. 2, pp. 74-78, August 2008.

[12] NASCOP \& NACC, "Kenya HIV County Profiles," Nairobi, 2014. 
[13] Joyce Kgatlwane et al., "Factors that facilitate or constrain adherence to antiretroviral therapy among adults at four public health facilities in Botswana: a pre-intervention study," 2006.

[14] Mathieu Rougemont, Beat E Stoll, Nadia Elia, and Peter Ngang, "Antiretroviral treatment adherence and its determinants in Sub-Saharan Africa: a prospective study at Yaounde Central Hospital, Cameroon," AIDS Research and Therapy, vol. 6, no. 21, October 2009.

[15] Karen S. Ingersoll and Jessye Cohen, "The impact of medication regimen factors on adherence to chronic treatment: a review of literature," Journal of behavioral medicine, vol. 31, no. 3, pp. 213-224, 2008.

[16] Walter H Curioso, Deanna Kepka, Robinson Cabello, Patricia Segura, and Ann E Kurth, "Understanding the facilitators and barriers of antiretroviral adherence in Peru: A qualitative study," BMC Public Health, vol. 10, no. 13, 2010.

[17] Camelia Protopopescu, François Raffi, Perrine Roux, Jacques Reynes, and Pierre Dellamon, "Factors associated with non-adherence to long-term highly active antiretroviral therapy: a 10 year follow-up analysis with correction for the bias induced by missing data," Journal of Antimicrobial Chemotherapy, vol. 64, no. 3, pp. 599-606, 2009.

[18] Bruce A Larson et al., "ART treatment costs and retention in care in Kenya: a cohort study in three rural outpatient clinics," Journal of the International AIDS Society, vol. 16, no. 1, p. 18026, 2013, doi: 10.7448/IAS.16.1.18026.

[19] Bentsi Sam Ophelia, "Factors That Constrain Adherence to Antiretroviral Therapy among HIV Positive Patients in the Sekondi-Takoradi Metropolis," School of Public Health, Kwame Nkrumah University of Science and Technology, Master's Thesis 2015.

[20] Leslie R Martin, Summer L Williams, Kelly B Haskard, and M Robin DiMatteo, "The challenge of patient adherence," Therapeutics and Clinical Risk Management, vol. 1, no. 3, pp. 189-199, 2005.

[21] Monica Malta, Maya L. Petersen, Scott Clair, Fernando Freitas, and Francisco I. Bastos, "Adherence to antiretroviral therapy: a qualitative study with physicians from Rio de Janeiro, Brazil," Cadernos de Saúde Pública, vol. 21, no. 5, pp. 1424-1432, 2005, http://dx.doi.org/10.1590/S0102-311X2005000500015.

[22] Daniel P. Kidder et al., "HIV Prevention in Care and Treatment Settings: Baseline Risk Behaviors among HIV Patients in Kenya, Namibia, and Tanzania," PLoS ONE, vol. 8, no. 2, p. e57215, February 2013.

[23] Heila E Aspeling and Neltjie Van Wyk, "Factors associated with adherence to antiretroviral therapy for the treatment of HIV-infected women attending an urban care facility," International Journal of Nursing Practice, vol. 14, no. 1, pp. 3-10, 2008, doi: 10.1111/j.1440-172X.2007.00659.x.

[24] Sarah Bott et al., "Rewards and challenges of providing HIV testing and counselling services: health worker perspectives from Burkina Faso, Kenya and Uganda," Health Policy Planning, pp. 1-12, 2014, doi: 10.1093/heapol/czu100.
[25] M. H. Chung et al., "A randomized controlled trial comparing the effects of counseling and alarm device on HAART adherence and virologic outcomes," PLoS Medicine, vol. 8, no. 3, p. e1000422, 2011.

[26] Sheri Weiser et al., "Barriers to Antiretroviral Adherence for Patients Living with HIV Infection and AIDS in Botswana," JAIDS Journal of Acquired Immune Deficiency Syndromes, vol. 34, no. 3, pp. 281-288, 2003.

[27] Lawrence Mbuagbaw et al., "Trends and determining factors associated with adherence to antiretroviral therapy (ART) in Cameroon: a systematic review and analysis of the CAMPS trial," AIDS Research and Therapy, vol. 9, no. 37, 2012, DOI: 10.1186/1742-6405-9-37.

[28] AIDSinfo, "Guidelines for the Use of Antiretroviral Agents in HIV-1-Infected Adults and Adolescents," Panel on Antiretroviral Guidelines for Adults and Adolescents, Department of Health and Human Services, 2016.

[29] Amsalu Alagaw, Wanzahun Godana, Mohammed Taha, and Tariku Dejene, "Factors Associated with Antiretroviral Treatment Adherence among Adult Patients in Wolaita Soddo Hospital," Journal of Tropical Diseases, vol. 1, no. 4, 2013.

[30] Ogbochi McKinney, Naomi N. Modeste, Jerry W. Lee, Peter C. Gleason, and Gisele Maynard-Tucker, "Determinants of Antiretroviral Therapy Adherence among Women in Southern Malawi: Healthcare Providers' Perspectives," AIDS Research and Treatment, vol. 2014, no. 2014, December 2014.

[31] Mariana Posse, Filip Meheus, Henri Van Asten, Andre Van Der Ven, and Rob Baltussen, "Barriers to access to antiretroviral treatment in developing countries: a review," Tropical Medicine \& International Health, vol. 13, no. 7, pp. 904-913, 2008, DOI: 10.1111/j.1365-3156.2008.02091.x.

[32] Sharada P. Wasti, Padam Simkhada, Julian Randall, Jennifer V. Freeman, and Edwin van Teijlingen, "Factors Influencing Adherence to Antiretroviral Treatment in Nepal: A Mixed-Methods Study," PLOS ONE, vol. 7, no. 5, p. e35547, 2012, http://dx.doi.org/10.1371/journal.pone.0035547.

[33] M Skovdal, C Campbell, K Nhongo, C Nyamukapa, and S Gregson, "Contextual and psychosocial influences on antiretroviral therapy adherence in rural Zimbabwe: towards a systematic framework for programme planners.," International Journal of Health Planning and Management, vol. 26, no. 3, pp. 296-318, 2011

[34] S Biadgilign, A. A Reda, and A Deribew, "Knowledge and attitudes of caregivers of HIV-infected children toward antiretroviral treatment in Ethiopia" Patient Education and Counseling, vol. 85, no. 2, pp. e89-e94, 2011.

[35] Beena Joshi et al., "Level of suboptimal adherence to first line antiretroviral treatment $\&$ its determinants among HIV positive people in India," The Indian Journal of Medical Research, vol. 140, no. 1, pp. 84-95, 2014.

[36] Kwasi E. Torpey et al., "Adherence Support Workers: A Way to Address Human Resource Constraints in Antiretroviral Treatment Programs in the Public Health Setting in Zambia," PLOS One, vol. 3, no. 5, pp. 1-9, 2008. 